\title{
Histologic evaluation of AlphaCor keratoprosthesis explanted following various complications
}

\author{
Nada Jiraskova ${ }^{a}$, Liliana Werner ${ }^{b}$, Nick Mamalis $^{b}$, Pavel Rozsival $^{a}$
}

\begin{abstract}
Aims. To report the histological findings in two AlphaCor artificial corneas explanted following complications,to compare the mechanism of biointegration and/or failure of the device and the correlation with clinical histories.

Methods. The first patient was diagnosed with optic deposits and corneal melting during the late postoperative period and required the removal of the device. The second patient experienced injury with penetration of the device 5 months after implantation. The explanted devices embedded within the corneal tissues were processed for pathological analysis at the John A. Moran Eye Center, University of Utah, Salt Lake City, USA. Histologic findings were compared with earlier histological results from animal studies and human case series and correlated with clinical histories.

Results. Both devices demonstrated normal skirt porosity. Light microscopic examination of the specimens disclosed adequate biointegration - colonization of the peripheral skirt with cellular elements present within the pores of the skirt. In the first patient (specimen 1) epithelial ingrowth was observed between the peripheral skirt and the posterior surface of the specimen, with partial extrusion of the device in that area. In the second case (specimen 2) the central, optic component of the device was missing.

Conclusion. Histologic findings of the AlphaCor skirt were consistent with earlier animal studies and human cases series reports. These findings confirm the occurrence of biointegration. Epithelial ingrowth and scattered chronic mononuclear inflammatory cells were observed in the area of partial extrusion in the patient with a history of melting prior to explantation.
\end{abstract}

Key words: AlphaCor, keratoprosthesis, biointegration, histology

Received: October 3, 2011; Accepted: April 4, 2012; Available online: June 1, 2012

http://dx.doi.org/10.5507/bp.2012.040

${ }^{a}$ Department of Ophthalmology, University Hospital, Sokolska 581, 50005 Hradec Kralove, Czech Republic

bJohn A. Moran Eye Center, University of Utah, 65 Mario Capecchi Drive, Salt Lake City, UT-84132, USA

Corresponding author: Nada Jiraskova, e-mail:jirasnad@fnhk.cz

\section{INTRODUCTION}

Keratoprosthesis devices consisting of a central optic and a biointegrating anchoring skirt have been developed to improve the outcomes of prosthetic corneal transplantation $^{1}$. AlphaCor is a biocompatible, flexible, one-piece synthetic cornea made from poly(2-hydroxyethyl methacrylate) (PHEMA) that has a peripheral region with interconnecting pores allowing biointegration with surrounding corneal tissue ${ }^{1,2}$. The two concentric regions are joined by means of an interpenetration of polymers across a junctional zone known as the interpenetrating polymer network (IPN) $\left(\right.$ ref. $\left.^{3}\right)$. The entire device has a diameter of $7.0 \mathrm{~mm}$, a thickness of $0.6 \mathrm{~mm}$ and surface curvatures that result in appropriate refractive power when implanted. The device is presently available in two powers: AlphaCor-A (for aphakic patients) and AlphaCor-P (for phakic or pseudophakic patients). The AlphaCor is implanted by means of a two-stage procedure. It is placed within a lamellar corneal pocket with tissue posterior to the optic being removed at the time of implantation (stage I surgery) and the tissue anterior to the optic being removed secondarily (stage II surgery). The porous skirt remains enclosed within corneal stromal tissue, with which it biointegrates due to cellular colonization and collagen deposition.

The most common complications encountered with the current devices include implant extrusion, stromal melting, surface spoilation and/or optic deposits, retroprosthetic membrane formation, infection, and glauco$\mathrm{ma}^{4.8}$.

The reported probability of retention rate with the AlphaCor device is $81 \%$ at one year and $60 \%$ at two years in a heterogeneous group of 377 patients $^{2}$. A device may require removal and replacement with donor tissue in the event of stromal inflammation, surrounding tissue melting and/or epithelial ingrowth. The other device non-related complication that requires explantation and donor penetrating keratoplasty ( $\mathrm{dPK})$ is trauma with penetration of the keratoprosthesis.

We present the histopathologic findings from two explanted AlphaCor artifical corneas, correlate them with clinical histories, and assess the adequacy of biointegration, making comparison with earlier animal studies and human cases series reports. 


\section{MATERIAL AND METHODS}

The AlphaCor device, inclusion criteria, surgical techniques and recommended management protocol have been described previously ${ }^{2}$. Both patients underwent the two-stage implantation procedure of artificial corneal surgery using the AlphaCor device.

First case, a 53 year old man was bilaterally blind for 20 years after serious chemical burn and multiple failed keratoplasties in both eyes. In September 2004 stage I of AlphaCor implantation was performed, and stage II was performed 3 months later (December 2004). The patient did well initially (from 1 month till 36 months after stage II surgery) with complete biointegration of the device into the surrounding host corneal rim, and apparently very good functional results $(0.4=6 / 15=20 / 50$ for distance and J.1 for near with correction). Deposits started to be observed on the optical surface of the device 2 years after implantation. Cleaning was performed 4 times using OPTI-FREE SUPRACLENS (Alcon Laboratories). In August 2008, acute melting with leakage was diagnosed and explantation was performed with a new corneal graft. The AlphaCor within the corneal button was fixated in $10 \%$ formalin inside a glass vial and submitted for histopathologic evaluation.

The second case, a 72 year old man had been bilaterally blind since 1970 after a serious alkali burn. He underwent unsuccessful keratoplasty and bilateral cataract surgery elsewhere. In March 2007 stage I of AlphaCor implantation and 3 months later stage II were performed. The patient gained a visual acuity of $0.25=6 / 24=20 / 80$ for distance and J. 4 for near with appropriate correction. The patient was excited and "returned to life", mainly to his big hobby from the past - tramping. Five months after AlphaCor implantation during one of his trips to the forest, he experienced a serious injury of his left eye with penetration of the device. Emergency penetrating keratoplasty was performed and the AlphaCor keratoprosthesis was replaced with a donor graft. Subluxated posterior chamber intraocular lens (PC IOL) was explanted within the capsular bag. Both specimens were fixated in $10 \%$ formaldehyde and sent for histopathological evaluation.

Each corneal button containing an AlphaCor keratoprosthesis was dehydrated, embedded in paraffin, and sectioned. Sections were stained with hematoxylin and eosin (H\&E), von Kossa method and alizarin red for detection of calcium, and Masson's trichrome (stains collagen in blue).

\section{RESULTS}

Specimen 1: The specimen consisted of a corneal button of $8.2 \mathrm{~mm}$ of diameter, containing an AlphaCor keratoprosthesis (KPro). The anterior corneal lamellae were opened in an area with a diameter of $4.7 \mathrm{~mm}$. White surface deposits were observed on the anterior optic surface of the device, forming an almost continuous crust on half of the optic periphery, with intervening clear areas.
A $2.3 \mathrm{~mm}$ diameter, eccentric opening in the posterior corneal lamellae was observed on the posterior surface of the specimen (Fig. 1).

Microscopic examination of multiple sections cut through the center of the specimen revealed that the peripheral skirt was well colonized, with cellular elements (foci of elements with dendritic pattern representing fibroblast-like cells) present within the pores of the skirt, including in the central skirt area. In one side of the sections (left side) epithelial ingrowth was observed between the peripheral skirt and the posterior surface of the specimen, which appeared to be partially extruded in that area. In the other side of the sections (right) the device skirt was apparently completely surrounded by corneal tissue. Scattered chronic mononuclear inflammatory cells were observed within the stroma of the corneal tissue surrounding the device in the left side of the sections (Fig. 2). Special stains for calcium (alizarin red and von Kossa's method) could not confirm the calcified nature of the superficial deposits in the sections (not shown).

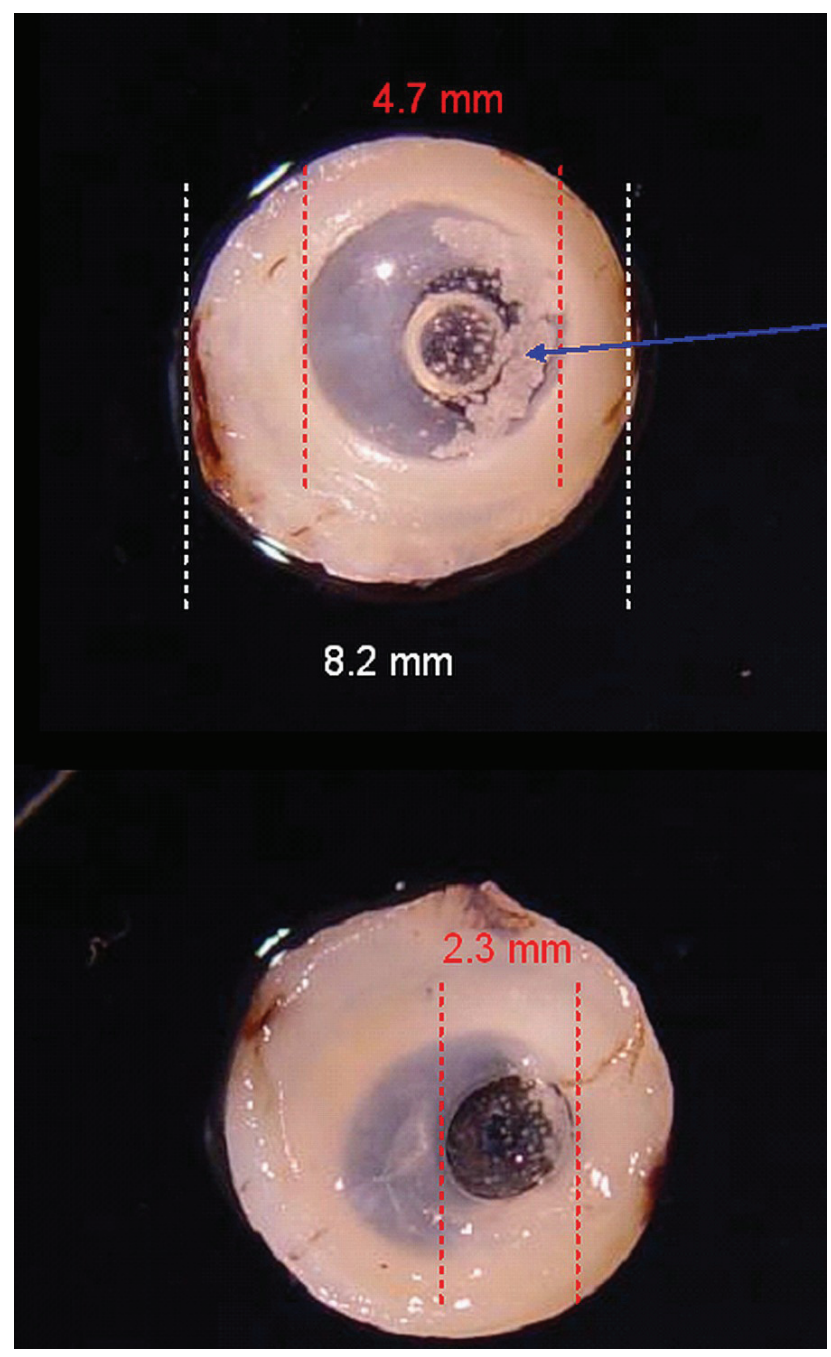

Fig. 1. Gross photographs of specimen 1. Top: Anterior surface. The arrow shows the surface deposits. Bottom: posterior surface. 

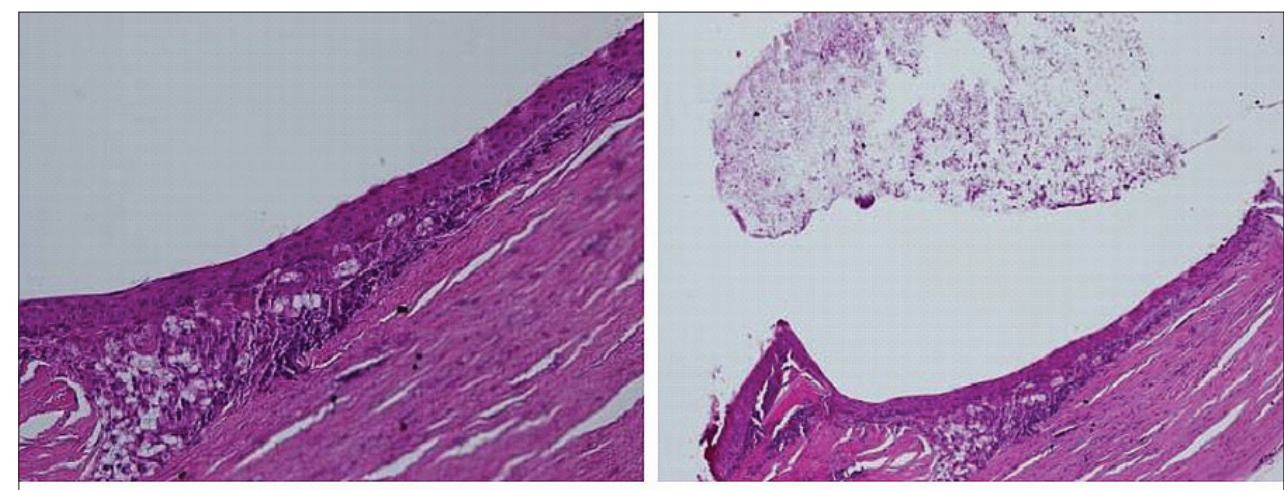

Fig. 2. Photomicrographs of specimen 1. Top: Left side of the section showing epithelial ingrowth. Bottom left: peripheral skirt with cellular elements. Bottom right: optic component. H \& E stain, original magnification X100, X200, and X400.
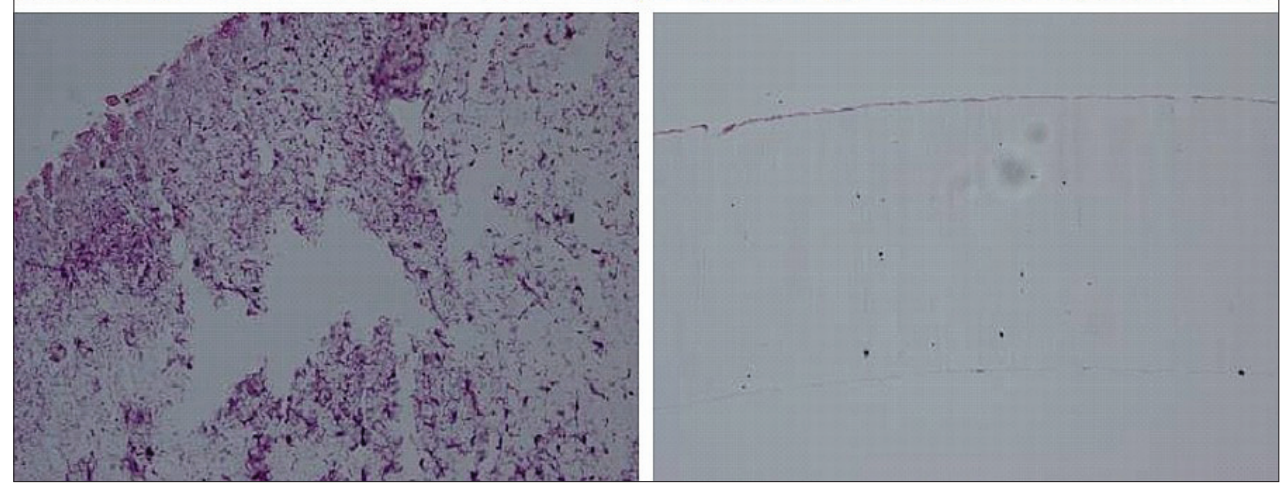

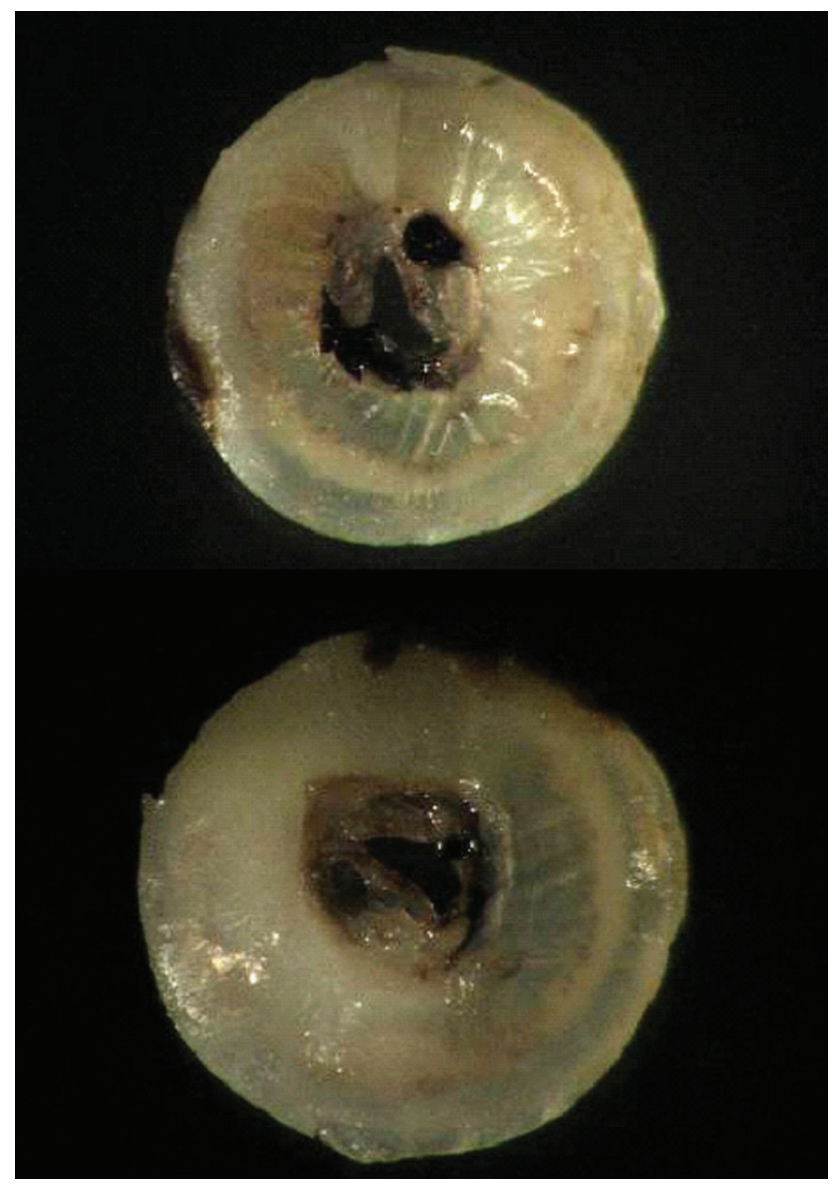

Fig. 3. Gross photographs of specimen 2. Top: Anterior surface. Bottom: posterior surface.
Specimen 2 A: The specimen consisted of a corneal button of $7.77 \mathrm{~mm}$ of diameter, with a thickness of 0.9 $\mathrm{mm}$, containing an AlphaCor keratoprosthesis. The diameter of the outer KPro skirt, implanted within the corneal tissue was $6.7 \mathrm{~mm}$. The optical part of the device appeared to be missing at the central area of the specimen, where a thin membrane was present. Fragments of iris tissue were found attached to the posterior aspect of the corneal button, at the center of the specimen (Fig. 3).

Microscopic examination of multiple histological sections obtained from the specimen revealed sagittal cuts of the keratoprosthesis, completely surrounded by corneal tissue. The central, optic component of the device was missing, and a membrane, composed of thin collagen fibers, vessels, and polymorphonuclear inflammatory cells was observed, making a bridge between the left and right side of the peripheral skirt. An acute, moderately severe inflammatory reaction was present within the corneal tissue at the extremities of this membrane. Fragments of iris tissue were present at the posterior aspect of the membrane. The corneal epithelium was found to be thin, with some areas of bullous keratopathy. Bowman's layer was absent. The stroma around the KPro exhibited mild vascularity. At the extremities of the sections (at the level of the sponge skirt), foci of elements with dendritic pattern were noted. They may represent fibroblast-like cells colonizing the pores of the material, and were especially present at the left side of the sections, including at the center of the sponge skirt (Fig. 4, 5).

The specimen 2B (not shown) consisted of a singlepiece IOL within the capsular bag. The lens was rigid in the dry state, and its overall design was consistent with a hydrophilic acrylic IOL (although the precise design/ model could not be identified). Direct light microscopic 

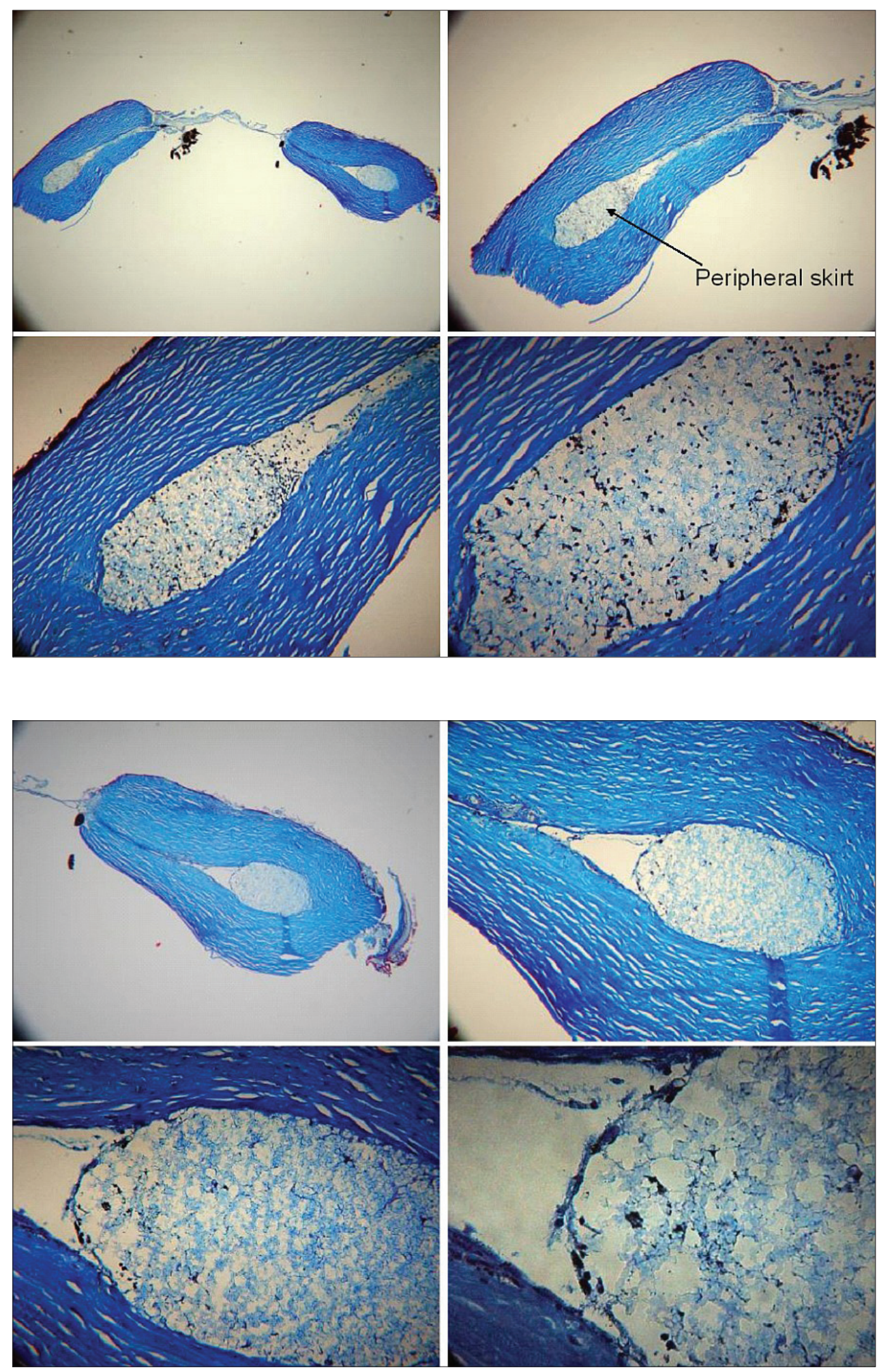

Fig. 4. Photomicrographs of specimen 2. Top left: overview. Top right and bottom: left side of the section, showing the cellular elements within the peripheral skirt of the device. Masson's trichrome stain, original magnification X20, X40, $\mathrm{X} 100$, and X200.

Fig. 5. Photomicrographs of specimen 2. Top left: overview. Top right and bottom: right side of the section, showing the cellular elements within the peripheral skirt of the device (there are less cells in comparison with the left side of the section). Masson's trichrome stain, original magnification X20, X40, X100, and X200. examination of specimen $2 \mathrm{~B}$ confirmed the presence of a single-piece IOL within the capsular bag, which exhibited a significant degree of contraction, and capsular folds. An opening of the capsular bag tissue was visible on the anterior surface of the specimen, which was consistent with a capsulorhexis of small diameter $(3 \mathrm{~mm})$. The tips of both haptic components kept contact with the optic component. Marks that could correspond to pits caused by Nd:YAG laser application were observed on the optic surface of the IOL. Iris pigment was present on the anterior surface of the specimen.

\section{DISCUSSION}

AlphaCor, earlier known as the Chirila KPro, was developed in an attempt to create a true alternative to human donor tissue in high-risk cases deemed ineligible 
for a regular donor corneal transplantation. Development of the Chirila KPro began at the Lions Eye Institute of Western Australia in 1989, and the current model of AlphaCor is the result of many years of laboratory and clinical research. The clinical trial phase was a period of learning and protocol development, and during this period a large quantity of data was published in peerreviewed journals ${ }^{9-15}$. The investigation process continues up to now, and ongoing collection of clinical results and histologic findings are important for a complete understanding of AlphaCor's performance and better management of those challenging cases.

The availability of histology from devices that were implanted in the earlier animal studies and the first trial phase of AlphaCor implantation in human allow us to compare the degree of biointegration and reexamine some of the fundamental assumptions made about the device based on these earlier results. It is important to assess whether the device retains its integrity in situ, whether biointegration occurs sufficient to ensure device fixation and prevent epithelial ingrowth, whether a stable noninflamed status is achieved within the device skirt, and the extent to which these processes are reduced or "reversed" if clinically evident stromal melting occurs.

The typical finding from early animal studies was of blood capillary invasion of the skirt, an initial inflammatory reaction, followed by fibroblastic cell invasion ensuring a firm skirt-stroma union. Over time, the number of inflammatory cells fell, and the proportion of viable over nonviable cells increased significantly, reflecting a normal, if slightly protracted, healing pattern ${ }^{13,14}$. Histologic examination of devices explanted from human subjects after complications demonstrated that AlphaCor sponge skirt allows biointegration by host fibroblastic cells, with deposition of collagen and with no evidence of calcification within the device skirts ${ }^{16}$. In both of our specimens the devices demonstrated normal skirt porosity, and the skirt was well colonized, with cellular elements (foci of elements with dendritic pattern representing fibroblastlike cells) present within the pores of the skirt, including in the central skirt area.

In the patient with penetrating injury (specimen $2 \mathrm{~A}$ ) the union between the skirt and surrounding corneal tissue was so firm, that penetration occurred in the central optic component and the force had also subluxated the IOL in the bag, but the anchoring biointegrating skirt of the device was not „pulled up“ from its bed.

In the patient with stromal melting and partial extrusion of the device, that had occurred 4 years after implantation, epithelial ingrowth was observed between the peripheral skirt and the posterior surface of the specimen 1 in the left side of the sections, which appeared to be partially extruded in that area. In the other side of the sections (right) the device skirt was apparently completely surrounded by corneal tissue. Scattered chronic mononuclear inflammatory cells were observed within the stroma of the corneal tissue surrounding the device in the side of the sections. This finding correlates with results of previous studies, where greater inflammatory cell populations in and around the device skirt were found in cases in which melting of the host stroma was observed ${ }^{15}$.

Another aspect of the response of the host tissue after KPro implantation is the production of enzymes. If this response is extensive, an increased risk of tissue melting and KPro loosening or extrusion would be expected. Coassin et al. ${ }^{1}$ have noted strong expression of various inflammatory cytokines, and only a few inflammatory cells in the three AlphaCor devices explanted due to corneal melting during the late postoperative period. They proposed that these cytokines were expressed by the keratocytes themselves because it is well known that the injured epithelial cells can stimulate fibroblast myodifferentiation of the keratocytes through inflammatory cytokines. Based on these observations, they speculated that an epithelial defect overlying the skirt portion of a well-biointegrated device can initiate the cascade of events and can lead to a corneal melt and device extrusion. In our first case (specimen 1) an epithelial ingrowth and scattered chronic mononuclear inflammatory cells were found in the area of partial extrusion of the device, and this finding supports this proposed elucidation of the mechanisms involved in the late failure of the device. Not only proper biointegration of the artificial material in the corneal stroma, but also achievement of complete and stable epithelization of the entire device is necessary for long-term retention of the AlphaCor KPro in situ.

Results from the animal studies ${ }^{16}$ suggested that biointegration was less extensive in alkali-burned rabbit corneas than healthy corneas. In contrast to this earlier finding, history of chemical injury was not a risk factor for corneal melt from clinical data, and also did not prevent biointegration in histologic evaluation of human explants ${ }^{15}$. The associations of preoperative factors and postoperative clinical occurrences are easier to determine than correlations with histologic findings in explants, as more clinical data are available than histologic results from explants. In our series the original diagnosis of serious chemical burn was the most common (47\%) and the clinical outcomes together with histopathologic finding of those two explants proved adequate biointegration. Optic deposition is the other main category of complication that relates to surface spoilation or deposition of substances within the hydrogel optic such that vision is reduced. The reported probability of intraoptic deposits is $8.4 \%$ in a heterogeneous group of 322 patients ${ }^{15}$. Several types of optic deposition and/or diffuse discoloration have been described. Brown intraoptic deposition occurred probably in association with smoking and/or environmental smoke. There are also some drugs (levobunolol, brimonidin) known to cause deposition in optic parts that should not be used in AlphaCor patients. White diffuse intraoptic deposition has previously been identified as calcium deposits within the gel $^{2,15}$. In our first case (specimen 1) white surface deposits were observed on the anterior optic surface of the device, forming an almost continuous crust on half of the optic periphery, with intervening clear areas. The deposits had morphological appearance of calcified deposits. However, this could not be confirmed with special 
stains for calcium, although it is possible that the sections did not pass through the area with deposits, or they were detached during tissue processing. It is noteworthy that in the other cases of calcification of the device evaluated in the past, the calcified deposits were within the optic substance of the device ${ }^{2,15}$.

\section{CONCLUSION}

In conclusion, the histopathologic findings from two explanted AlphaCor devices were in good correlation with clinical histories of both patients. Although AlphaCor may be a feasible substitute for donor corneas in selected cases, both the surgeons and the patients should be aware of the possible complications.

\section{ACKNOWLEDGEMENT}

Supported in part by research project MZO 00179906 from the Ministry of Health, Prague, Czech Republic.

\section{CONFLICT OF INTEREST STATEMENT}

The authors stated that there are no conflicts of interest regarding the publication of this article.

\section{REFERENCES}

1. Coassin M, Zhang C, Green WR, Acquavella JV, Akpek EK Histopathologic and Immunologic Aspects of AlphaCor Artificial Corneal Failure. Am J Ophthalmol 2007;144:699-704.

2. Hicks CR, Crawford GJ, Dart JKG, Grabner G, Holland E, Stulting RD, Tan DT, Bulsara M. AlphaCor Clinical Outcomes Cornea 2006;25:1034-42.
3. Chirila TV, Vijayasekaran S, Horne R, Interpenetrating polymer network (IPN) as a permanent joint between the elements of a new type of artificial cornea. J Biomed Mater Res 1994;28:745-53.

4. Hicks CR, Chirila TV, Werner L ,Crawford GJ, Apple DJ, Constable IJ. Deposits in artificial corneas: risk factors and prevention. Clin Experiment Ophthalmol 2004;32:185-91.

5. Chirila TV, Morrison DA, Hicks CR, Gridneva Z, Barry CJ, Vijayasekaran, $\mathrm{S}$. In vitro drug-induced spoliation of a keratoprosthetic hydrogel. Cornea 2004;23:620-9.

6. Hicks CR, Hamilton S. Retroprosthetic membranes in AlphaCor patients: risk factors and prevention. Cornea 2005;24:692-8.

7. Netland PA, Terada H, Dohlman CH. Glaucoma associated with keratoprosthesis. Ophthalmology 1998;105:751-7.

8. Nouri M, Terada H, Alfonso EH, Foster CS, Durand ML, Dohlman CH. Endophthalmitis after keratoprosthesis: incidence, bacterial causes, and risk factors. Arch Ophthalmol 2001;119:484-9.

9. Chirila TV, Thompson-Wallis DE, Crawford GJ, Constable IJ, Vijayasekaran S. Production of neocollagen by cells invading hydrogel sponges implanted in the rabbit cornea. Graefes Arch Clin Exp Ophthalmol 1996;234:193-8.

10. Fitton JH, Ziegelaar BW, Hicks CR, Clayton AB, Crawford GJ, Constable $\mathrm{IJ}$, Chirila TV. Assessment of anticollagenase treatments after insertion of a keratoprosthetic material in the rabbit cornea. Cornea 1998;17:108-14.

11. Hicks $C R$, Chirila TV, Clayton $A B$, Fitton JH, Vijayasekaran S, Dalton PD, Lou X, Platten S, Ziegelaar B, Hong Y, Crawford GJ, Constable IJ. Clinical results of implantation of the Chirila keratoprosthesis in rabbits. Br J Ophthalmol 1998;82:18-25.

12. Hicks CR, Chirila TV, Dalton PD, Clayton AB, Vijayasekaran S, Crawford GJ, Constable IJ . Keratoprosthesis: preliminary results of an artificial corneal buton as a full-thickness implant in the rabbit model. Aust N Z J Ophthalmol 1996;24:297-303.

13. Vijayasekaran S, Hicks CR, Chirila TV, Fitton JH, Clayton AB, Lou $X$, Platten ST, Crawford GJ, Constable IJ. Histologic evaluation during healing of hydrogel core-and-skirt keratoprostheses in the rabbit eye. Cornea 1997;16:352-9.

14. Vijayasekaran S, Fitton JH, Hicks CR, Chirila TV, Crawford GJ, Constable IJ. . Cell viability and inflammatory response in hydrogel sponges implanted in the rabbit cornea. Biomaterials 1998;19:225567.

15. Hicks CR, Werner L, Vijayasekaran S, Mamalis N, Apple DJ. Histology of AlphaCor skirts: evaluation of biointegration. Cornea 2005;24:93340.

16. Hicks CR, Vijayasekaran S, Chirila TV, Platten ST, Crawford GJ, Constable IJ. Implantation of PHEMA keratoprostheses after alkali burns in rabbit eyes. Cornea 1998;17:301-8. 